\title{
A COMPUTATIONAL MODEL FOR HIGH CURRENT DENSITY ARC PLASMAS
}

\author{
M. Alija*, M. Kurrat \\ Faculty of Electrical Engineering, Information Technology, Physics, Technische Universität Braunschweig, \\ 38106 Braunschweig, Germany \\ * m.alija@tu-braunschweig.de
}

\begin{abstract}
A computational model for high current density arc plasmas is developed. Under the assumption of thermodynamic equilibrium the arc plasma is described as a compressible laminar fluid based on the magnetohydrodynamic (MHD) equations and the transport and thermodynamic properties of air. The arc plasma is studied in time and space on macroscopic values such as the temperature and the pressure. The simulation results are discussed and future research work is identified addressing the scientific domain of high current density arc plasmas.
\end{abstract}

Keywords: computational plasma physics, high current density arc plasma, magnetohydrodynamics.

\section{Introduction}

Computational models are essential for the understanding of the physics of arc plasmas. To encapsulate the behaviour of arc plasmas in time and space several physical domains are addressed in the computational models. Promising models studying high current densities arc plasmas are embraced in [1-3]. The arc plasma is described as a single fluid using the MHD equations and studied in time and space. A key parameter for the development of the models is the current density. The coupling and the influcence of the magnetic field is addressed in $[2,3]$ for current densites of $j \approx 10^{6} \mathrm{~A} \cdot \mathrm{m}^{-2}$. In [1] the focus lies on the influence of the heat transfer by radiation for a current density of $j \approx 10^{9} \mathrm{~A} \cdot \mathrm{m}^{-2}$. Investigations of [4] shows even higher current densities reaching values of approximately $j \approx 10^{11} \mathrm{~A} \cdot \mathrm{m}^{-2}$ in the arc plasma fall regions. Higher current densities leads to higher energy densities in the plasma and subsequently to a wider range of operating conditions. For this the phenomena in an arc plasmas are still not fully understood. To advance the understanding and motivated by the studies [1-3], in this paper a computational model for high current density arc plasmas for $j=1 \cdot 10^{8} \mathrm{~A} \cdot \mathrm{m}^{-2}$ is presented. Starting from the Boltzmann equation the computational model is presented. The arc plasma is studied and future research work is identified.

\section{Computational model}

Starting from Liouville theorem the development of the distribution of the species in a plasma is described by the generalized Boltzmann equation $[2,3,5,6]$ with

$$
\frac{\partial f}{\partial t}+\mathbf{v} \cdot \frac{\partial f}{\partial \mathbf{x}}+\frac{q}{m}(\mathbf{E}+\mathbf{v} \times \mathbf{B}) \cdot \frac{\partial f}{\partial \mathbf{v}}=\sum C(f)
$$

Covering the interaction of the species as elastic or inelastic collisions by $C(f)$ and the influence of elec- tromagnetic fields by $\mathbf{E}$ and $\mathbf{B}, f$ presents the distribution of the species, where $\mathbf{x}$ is the spatial and $\mathbf{v}$ is the velocity phase space coordinate. This leads to a six dimensional description of the species. Using the moments of equation 1 the MHD equations are derived, which describes the arc plasma as a fluid with electrons and ions. Under the assumption of thermal equilibrium and quasi neutrality the one-fluid model is derived. The plasma is considered as a compressible and laminar flow, where the electrons and ions are not separated. The equations of the computational model are expressed in a conservative form, where the behaviour of the mass and energy is of importance. The conservation of mass is expressed by $[1,3,5,7]$

$$
\frac{\partial \rho}{\partial t}+\nabla \cdot(\rho \mathbf{v})=0
$$

where $\rho$ is the density and $\mathbf{v}$ the velocity. As the density of the plasma can change with pressure, the time derivative of the density is considered in the equation. The modeling of the mass is supplemented by formulating the equation of the impuls for the particles with $[1,3,5,7]$

$$
\frac{\partial \rho \mathbf{v}}{\partial t}+\rho(\mathbf{v} \cdot \nabla) \mathbf{v}=\nabla \cdot(\boldsymbol{\tau}-p \mathbf{I})+\mathbf{j} \times \mathbf{B}
$$

where $\tau$ the viscous tensor, $p$ is the pressure, $\mathbf{I}$ the identity tensor, $\mathbf{j}$ the electric current density and $\mathbf{B}$ the magentic field. A weak coupling is performed by implementing the crossproduct of $\mathbf{j}$ and $\mathbf{B}$. This sets of equations are extended by formulating the conservation for the energy with $[3,5]$

$$
\rho c_{p}\left(\frac{\partial T}{\partial t}+\mathbf{v} \cdot \nabla T\right)=\nabla \cdot(\lambda \nabla T)+(\mathbf{j} \cdot \mathbf{E})-W_{\mathrm{R}}
$$

where the weak coupling is implemented by the Joule heating per unit volume by the product of the current density $\mathbf{j}$ and electric field strength $\mathbf{E} . c_{p}$ is the specific 
heat capacitance, $T$ the temperature, $\lambda$ the thermal conductivity and $W_{\mathrm{R}}$ the radiation losses per unit volume. Pressure and visocus work are considered as negligible small. The electromagnetic fields are described by using Maxwell's equations. Assuming quasistatic behaviour, the electric potential is calculated by the scalar definiton of $\phi$ as a Laplace equation with

$$
\nabla \cdot(\kappa \nabla \phi)=0
$$

By using the electric potential $\phi$ the electric field is solved using $\mathbf{E}=-\nabla \phi$ and the electric flow field is calculated by $\mathbf{j}=-\kappa \nabla \phi$. The magnetic field is modeled with a further differential equation using the magnetic vectorpotential. The vectorpotential is formulated as a Poisson equation with

$$
\Delta \mathbf{A}=-\mu_{0} \mathbf{j}
$$

where each component of $\mathbf{A}$ is calculated by the current density $\mathbf{j}$. With the vector potential the magnetic field is solved by

$$
\mathbf{B}=\nabla \times \mathbf{A}
$$

The heat distribution in the electrodes is modeled by Fourier's law. For this the differential equation with

$$
\rho_{\text {Elec }} c_{\text {Elec }} \frac{\partial T}{\partial t}=\nabla \cdot\left(\lambda_{\text {Elec }} \nabla T\right)+\left(\kappa_{\text {Elec }} \mathbf{E}^{2}\right)
$$

is solved for the electrodes. The properties of the electrodes in equation 8 are selected for copper [8], with the $\rho_{\text {Elec }}=8900 \mathrm{~kg} \cdot \mathrm{m}^{-3}$ as the density, $c_{\text {Elec }}$ is the specific heat capacitance with $385 \mathrm{~J} \cdot(\mathrm{kg} \cdot \mathrm{K})^{-1}$ and $\lambda_{\text {Elec }}$ the thermal conductivity with $400 \mathrm{~W} \cdot(\mathrm{m} \cdot \mathrm{K})^{-1} \cdot \kappa_{\text {Elec }}$ is the electrical conductivity with $5.99 \mathrm{MS} \cdot \mathrm{m}^{-1}$. Energy transport by radiation in the plasma is described by the net emission coefficient $W_{\mathrm{R}}$ as it offers an acceptable computational performance and describes the physical process of absoprtion and emission in the arc plasma. $W_{\mathrm{R}}$ is derived by building the divergence of the heat flux [9] and modeling the arc plasma as a cylindrical isothermal channel with a radius $r$. By using this assumption $W_{\mathrm{R}}$ is implemented by

$$
W_{\mathrm{R}}=\int_{0}^{\infty} B_{f} \kappa_{f} e^{\left(-\kappa_{f} r\right)} \mathrm{d} f
$$

where $B_{f}$ is the intensity of a black body radiator according to Planck and $\kappa_{f}$ the spectral absorption coefficient. The calculated values for $W_{\mathrm{R}}$ in dependence of $T$ and $p$ are used based on $[10,11]$. The computational model is solved by using the finite element algorithm of Comsol Multiphysics.

\section{Computational geometry, boundary and initial conditions}

In this part the geometry and the boundary conditions of the computational model are presented. The selected geometry is shown in Figure 1. The calculation is performed in $2 \mathrm{D}$ in order to improve the computational time and to encapsulate the physical compelxities. The plasma chamber has width of $25 \mathrm{~mm}$ and a height of $40 \mathrm{~mm}$. The electrode A and electrode B are $10 \mathrm{~mm} \times 15 \mathrm{~mm}$. The high density arc plasma is ignited between the electrodes and evolves in the chamber.

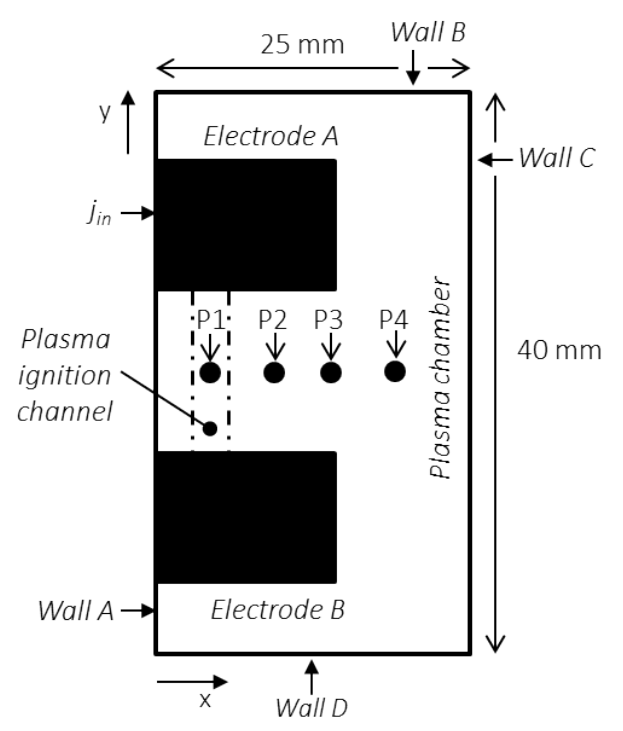

Figure 1. 2D geometry for which the computational model is solved

A high current density with $j_{\text {in }}=1 \cdot 10^{8} \mathrm{~A} \cdot \mathrm{m}^{-2}$ is set to electrode A by implementing $j_{\text {in }}=\mathbf{n} \cdot \mathbf{j}$. For a $1 \mathrm{~mm}$ thick electrode this corresponds to a $1 \mathrm{kA}$ current. The boundaries of the plasma chamber are implemented with the condition $\mathbf{n} \cdot \mathbf{j}=0$. For the boundaries at the interface between the electrodes and the plasma, continuity is implemented for the normal component of $\mathbf{j}$. The tangential component of $\mathbf{j}$ at the interface is formulated by the ratio of $\kappa$ of the electrodes and the air plasma. For the electrode $\mathrm{B}$ the potential is fixed to $\varphi=0$. The magnetic field is initially set to $\mathbf{B}=\mathbf{0}$. It is assumed that the magnetic field decreases at the boundaries of the plasma chamber nearly to zero. This is implemented by building the cross-product of $\mathbf{n} \times \mathbf{A}=\mathbf{0}$ for the boundaries of the plasma chamber. The temperature of the plasma chamber and the electrodes is set initially to $T=293.15 \mathrm{~K}[12]$. The pressure in the plasma chamber is initially defined with $p=101325 \mathrm{~Pa}$ [12]. The initial temperature for the plasma ignition is set to $T \approx 15 \mathrm{kK}$, as the electrical conductivity $\kappa(T, p)$ is nearly constant and can be simplified in this way. The evolvment of the plasma in the chamber is implemented by setting the heat flux at the boundaries of the chamber to $\mathbf{n} \cdot \mathbf{q}=0$. A movement of the plasma along the electrodes and the walls is assumed to be zero and implemented by setting the boundaries of the electrodes and the walls to $\mathbf{v}=\mathbf{0} \mathrm{m} \cdot \mathrm{s}^{-1}$. The plasma velocity field is implemented initially with $v_{x}=0 \mathrm{~m} \cdot \mathrm{s}^{-1}$ and $v_{y}=0 \mathrm{~m} \cdot \mathrm{s}^{-1}$. 


\section{Air plasma properties}

The computational model is completed by the physical properties of an air plasma. They can be divided into the thermodynamic properties and transport properties. The density $\rho(T, p)$, the specific heat capacitance $c_{\mathrm{p}}(T, p)$ and the adiabatic coefficient $a c(T, p)$ belong to the thermodynamic properties. The electrical conductivity $\kappa(T, p)$, the thermal conductivity $\lambda(T, p)$ and the dynamic viscosity $\mu(T, p)$ belong to the transport properties. The properties are calculated for a wide temperature range of up to $100 \mathrm{kK}$ and a wide pressure range of up to 123 bar based on [13]. In Figure 2 the electrical conductivity over the temperature for three different pressures is depicted.

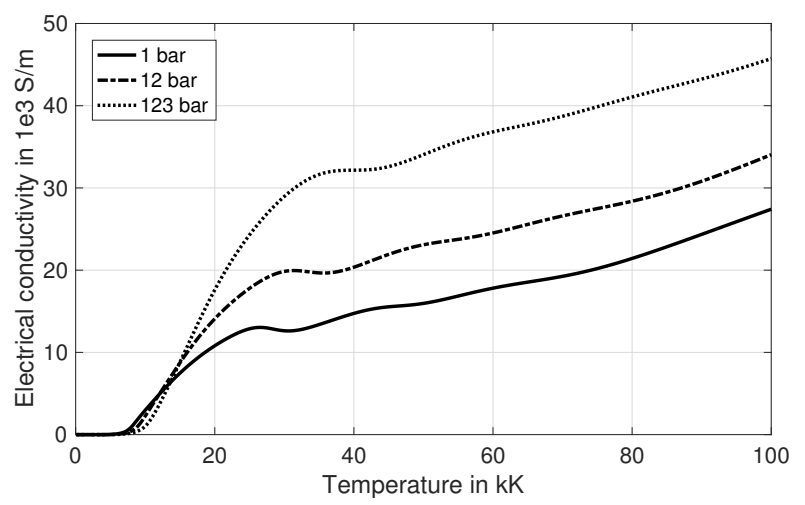

Figure 2. Electrical conductiviy $\kappa(T, p)$ of an air plasma in dependence of the temperature and the pressure

\section{Computational experiments}

In this part the results of the computational experiments are presented. For the calculation times with $t=5 \mu \mathrm{s}, 10 \mu \mathrm{s}, 20 \mu \mathrm{s}$ and $30 \mu \mathrm{s}$ the distribution of the temperature is depicted in Figure 3.

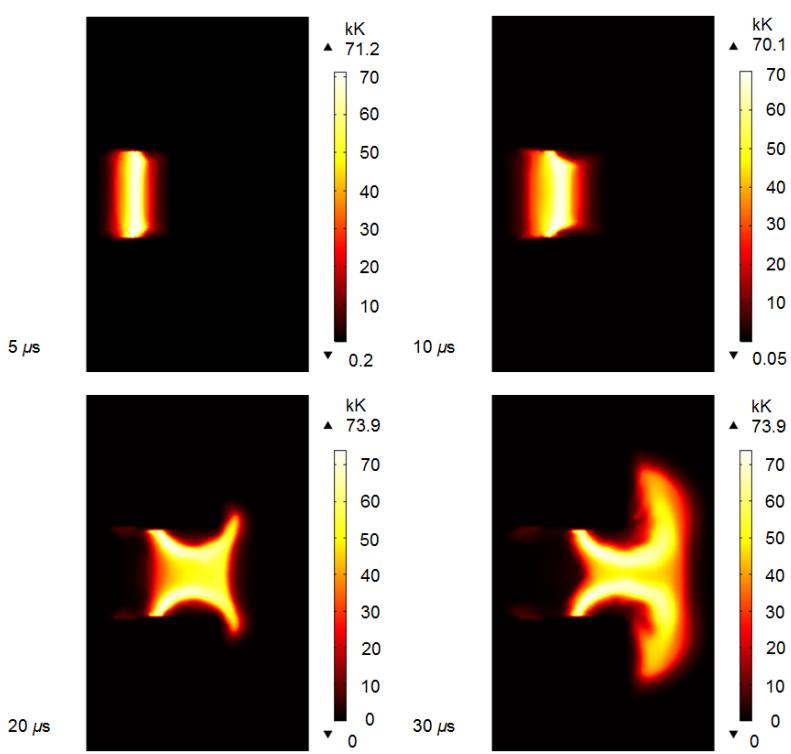

Figure 3. Distribution of the plasma temperature for selected calculation times.

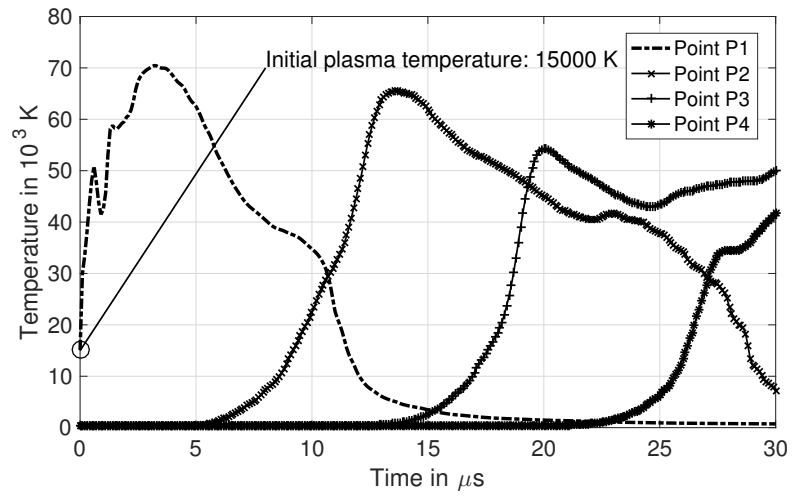

Figure 4. Temperature behaviour of the plasma in dependence of the time.

The energy, that is fed into the plasma chamber by Joule heating per unit volume, can be associated with a change of the physical state values of the air plasma. Considering the temperature distribution in Figure 3, the plasma is heated up. Starting from the ambient temperature, a temperature increase can be observed in Figure 3 ranging up to 73923 K. For $t=5 \mu \mathrm{s}$ the temperature increase is concentrated in the plasma channel. For $t=10 \mu \mathrm{s}, 20 \mu \mathrm{s}$ and $30 \mu \mathrm{s}$ the temperature increase encompasses a wider area in the plasma chamber. In order to study the distribution of plasma in detail, four evaluation points along the positive $\mathrm{x}$ axis are selected. The selected points are marked in Figure 1 with P1, P2, P3 and P4. The calculated temperatures over the time for the points are depcited in Figure 4. For each of the selected points the temperature shows a similiar behaviour.

As a consequence of the Joule heating the temperature raises continously till to a maximum value. For $\mathrm{P} 1$ the maximum is $T_{\max } \mathrm{P} 1=70393 \mathrm{~K}$. After $T_{\max }$ the temperature decreases continously to the intial temperature $T=293.15 \mathrm{~K}$. Compared to P1, the temperature increase for P2, P3 and P4 in Figure 4 starts with delay. This can be associated with a movement of the plasma in the plasma chamber along the positive $x$ axis. The movement of the plasma can also be observed in the distribution of the temperature in Figure 3. In order to study the plausibility of the computational experiments, the pressure and density behviour over the time are considered. For the selected points the pressure is depicted in Figure 5.

Comparing the pressure and temperature curve for P1 a proprotional behaviour can be observed as expected. For P1 a maximum pressure with $p_{\max , \mathrm{P} 1}=904.5 \mathrm{kPa}$ is calculated. After $p_{\max }$ the pressure decreases below the initial pressure. This can be explained by the increase of $T$ and $p$ along the selected points. The pressure curves for P2, P3 and $\mathrm{P} 4$ show a steeper increase compared to the temperature curves in Figure 5. This is a result of the reflected pressure at the chamber wall A. In Figure 6 the calculated densities for the selected points over the time are shown. For a given pressure, the densities 


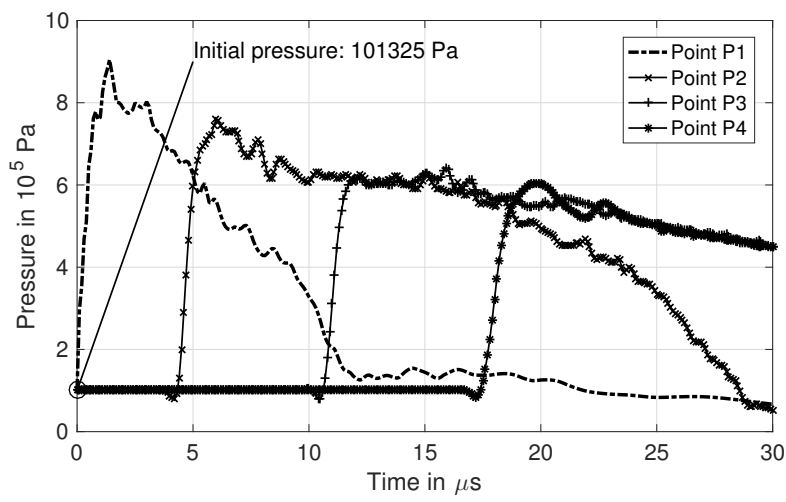

Figure 5. Pressure behaviour of the plasma in dependence of the time

in Figure 6 show an antiproprotional behaviour to the temperature curves in Figure 4 as expected.

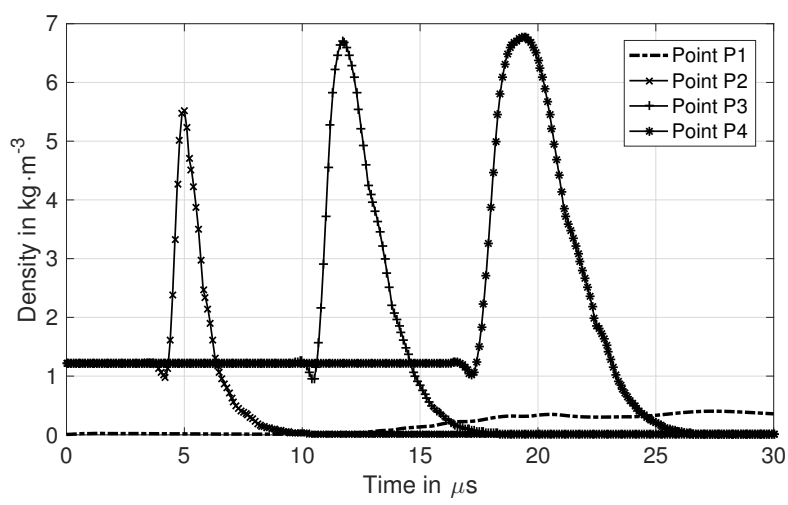

Figure 6. Density of the plasma in dependence of the time for the selected points

Considering the temperature and pressure curve for example for $\mathrm{P} 2$ for $t \leq 5 \mu \mathrm{s}$, ambient conditions with $T=293.15 \mathrm{~K}$ and $p=101325 \mathrm{~Pa}$ are given at this point in the plasma chamber. The density for this point at the same time is $\rho=1.217 \mathrm{~kg} \cdot \mathrm{m}^{-3}$, which is approximately the density of air with $\rho=1.293 \mathrm{~kg} \cdot \mathrm{m}^{-3}$ [14] at ambient conditions. The computational results seem to be generally plausible, when comparing the results qualitatively with the empirical model of an ideal gas [14], where $T \sim p$ and $T \sim 1 / \rho$.

\section{Conclusions and future work}

A computational model for high current density arc plasmas is developed and the computational experiments are presented. With the derived equations a high current density arc plasma is simulated for a $2 \mathrm{D}$ geometry. Plausibility of the computational results is fostered by the qualitative comparison with an empirical model. Further investigations are motivated, addressing the modeling of the plasma and electrode interaction and the description of the plasma ignition. For this wide temperature range attention should be also paid to the radiation, which is evident when considering the Stefan-Boltzmann law. Furthermore a quantitative validation of the computational results should be addressed.

\section{References}

[1] C. Sander, J.-E. Schmutz, and M. Kurrat. Analysis for radiation discretization for modelling spark gap for surge currents. Plasma Physics and Technology, 4(1):56-59, 2017. doi:10.14311/ppt.2017.1.56.

[2] P. Huguenot. Axisymmetric high current arc simulations in generator circuit breakers based on realgas magnetohydrodynamics models. ETH Zurich, Zurich, 2008. doi:10.3929/ethz-a-005579457.

[3] M. Kumar. Three dimensional high current arc simulations for circuit breakers using real gas resistive magnetohydrodynamics. ETH Zurich, Zurich, 2009. doi:10.3929/ethz-a-005927654.

[4] S. Coulombe and J. Meunier. Arc-cold cathode interactions: parametric dependence on local pressure. Plasma Sources Science and Technology, 6(4):11, 1997. doi:10.1088/0963-0252/6/4/008.

[5] J. H. Ferziger and M. Perić. Numerische Strömungsmechanik. 3. Springer, 2008.

[6] H. Goedbloed and S. Poedts. Principles of Magnetohydrodynamics. Cambridge University Press, 2004.

[7] F. F. Chen. Introduction to Plasma Physics and Controlled Fusion. Springer International Publishing, 2016.

[8] E. Ivers-Tiffée and W. von Münch. Werkstoffe der Elektrotechnik. B.G. Teubner Verlag, 2007.

[9] M. F. Modest. Radiative Heat Transfer. Academic Press, Elsevier, 2013.

[10] Y. Naghizadeh-Kashani, Y. Cressault, and A. Gleizes. Net emission coefficient of air thermal plasmas. Journal of Physics, 35(2925):10, 2002. doi: 10.1088/0022-3727/35/22/306.

[11] T. Billoux, Y. Cressault, P. Teulet, and A. Gleizes. Calculation of the net emission coefficient of an air thermal plasma at very high pressure. Journal of Physics, 406(012010):11, 2012. doi:10.1088/1742-6596/406/1/012010.

[12] P. J. Mohr, D. B. Newell, and B. N. Taylor. Codata recommended values of the fundamental physical constants: 2014. Journal of Physical and Chemical Reference Data, 45(4):74, 2016. doi:10.1063/1.4954402.

[13] D. Angola, G. Colonna, C. Gorse, and M. Capitelli. Thermodynamic and transport properties in equilibrium air plasmas in a wide pressure and temperature range. European Physical Journal, 46:129-150, 2008. doi:10.1140/epjd/e2007-00305-4.

[14] M. Bartelmann, B. Feuerbacher, T. Krüger, D. Lüst, A. Rebhan, and A. Wipf. Theoretische Physik 4 Thermodynmaik und Statistische Physik. Springer Spektrum, 2018. 